\title{
ESTRATEGIAS DEL ARTE PARTICIPATIVO: ENTRE LA MICRO-POLÍTICA Y EL SOFTWARE SOCIAL
}

\author{
Cuautli Exal Martínez Sánchez \\ Universidad Politécnica de Valencia. Doctorando
}

\section{Resumen}

La hibridación de las tecnologías digitales con la totalidad de las actividades humanas ha desencadenado una transformación irreversible en múltiples aspectos de nuestra vida cotidiana pero sobre todo, en los procesos de construcción sociocultural; Lo efímero, híbrido, virtual, interactivo, heterogéneo, son ejes entorno a los cuales construimos nuevas relaciones sociales. Material de análisis y reflexión para el desarrollo de las prácticas artísticas contemporáneas. En este sentido, las estrategias participativas impulsadas desde las prácticas artísticas desarrollan estructuras de relación, espacios de encuentro, diálogo y autoproducción colectiva. Podríamos afirmar que exploran el campo de las relaciones sociales (la conectividad social) y sus potenciales expresivos, narrativos, políticos, poéticos, estéticos, etc. Este artículo propone un análisis de las estrategias artísticas participativas como herramientas híbridas e interdisciplinares que propician a través de nuevas metodologías de investigación, la reflexión sobre nuevos modos de producir subjetividad y visualidad, producción social y emancipación cultural.

\section{Palabras-clave: ARTE PARTICIPATIVO; SOFTWARE SOCIAL;TECNOLOGÍAS BLAN-} DAS; ARTIVISMO; ESTRATEGIAS MICROPOLÍTICAS

\section{STRATEGIES OF PARTICIPATORY ART: BETWEEN MICRO-POLICY AND SOCIAL SOFTWARE}

\section{Abstract}

Hybridization of digital technologies with all human activities has triggered an irreversible transformation in many aspects of our daily lives but especially in sociocultural construction processes; ephemeral, hybridity, virtuality, interactivity, heterogenity, disruptiveness, are axes around which we build new social relations. Matter of analysis and reflection in the developing of contemporary art practices. In this sense, participatory strategies promoted by artistic practices develop relationship structures, spaces for encounter, dialogue, common action and collective self-production. We could say that these artistic practices explore the field of social relations (social entanglement and engagement) and their expressive, narrative, politic, poetic or aesthetic, potentials. This article proposes an analysis of participatory artistic strategies as hybrid and interdisciplinary tools that lead through new research methodologies to new ways of producing subjectivity and visuality, social production and cultural emancipation.

\section{Keywords: PARTICIPATORY ART; SOCIAL SOFTWARE; SOFT TECHNOLOGIES; MICROPOLITIC STRATEGIES}

\footnotetext{
Martínez Sánchez, Cuautli Exal. 2016. “Estrategias del arte participativo: Entre la micro-política y el software social”. AusArt 4(1): pp-pp. 207-216 D0I: 10.1387/ausart.16704
}

\section{AUSART}




\section{INTRODUCCIÓN}

La capa informática de nuestra civilización, focalizada en la "nube", se ha convertido en factor determinante de la construcción de nuestra realidad. Desde la aparición de las primeras máquinas lógicas (militares) a principios de los años cuarenta del siglo pasado, el desarrollo de las tecnologías computacionales y de telecomunicación ha propiciado un nuevo escenario de organización global; nuevos modelos políticos, económicos, culturales, sociales. Basamos nuestras relaciones en nuevos ejes constructivos como la interconexión, complejidad, globalidad, heterogeneidad, multi-territorialidad, relatividad, interactividad, etc. podríamos afirmar que se ha producido una profunda transformación en la construcción cultural, como menciona José Luis Brea $(2007,7)$ : "Un cambio fundamental en el concepto de 'cultura' es que ya no es principalmente herramienta de almacenamiento y consignación patrimonial, archivística, sino sobre todo dinámica, proceso y arquitectura relacional. Herramienta de interacción y principio de la acción comunicativa".

La hibridación tecnología-comunicación-cultura, y sus procesos (globales y locales) nos sitúan ante un hecho inapelable sobre nosotros mismos; no es posible comprendernos como sociedad contemporánea sin la tecnología incorporada en todos los actos cotidianos, es una "segunda piel", un segundo lenguaje que nos constituye y nos transforma. Dicha transformación tiene que ver con las formas técnicas en las que la comunicación contemporánea se produce, codifica y disemina a nivel global. Las formas a las que accedemos a la información y las dinella. Sin embargo, la transformación es bidireccional; si bien la "capa informática" afecta la "capa cultural" de la comunicación contemporánea (Manovich 2005, 93). Cuando las tecnologías computacionales entran en contacto con las ciencias sociales como es el caso del arte, también se ven afectadas. Existe una reinterpretación de lenguajes y códigos, una transformación semántica y una resignificación de las funciones y métodos lógicos, cuantitativos e informáticos. Las estrategias participativas, exploran la producción de nuevos dispositivos tecnológicos de relación social, donde las variables subjetivas, culturales y contextuales que afectan los procesos tecnológicos, terminan infiriendo en el enfoque de su producción.

Los trabajos participativos realizados en el ámbito artístico producen espacios de encuentro, dispositivos de acción, redes de comunicación-interacción, diálogos tecno-culturales, que pueden resultar efectivos en la construcción de nuevos mecanismos sociales de acción y producción colectiva. Cabe destacar que su impacto más relevante se produce a nivel comunicacional; 
estos trabajos artísticos muestran formas distintas de relación, interacción y acción comunitaria, diseñan estructuras de relación por medio de las cuales los participantes, construyen mediante su acción y experiencia, ejercicios de "micro-emancipación". Estas prácticas interactivas cobran relevancia en la construcción social contemporánea, pues diseñan momentos divergentes de relación. "La activación del espectador es un acto emancipador en tanto que es análogo a la participación del espectador en el mundo. Una relación transitiva viene implícita entre 'espectador activo' y la participación activa en el ámbito socio-político" (Bishop 2005, 36).

El concepto de participación en el arte no es nuevo, hay registros ya en los años veinte, de compañías teatro soviético del Proletkult donde cada individuo dentro de la compañía, desde la gente que montaba escenario, hasta los actores, participaban en todos los aspectos de la producción; diseño de vestuario, construcción de la escenografías, hasta la dirección de las escenas o la creación de nuevas obras. A principios de los años treinta las obras de radioteatro desarrolladas por Bertolt Brecht buscaban estrategias, para romper con la unidireccionalidad de la comunicación radial y establecer grados de participación entre los radioescuchas y el desarrollo de las tramas narrativas de sus obras (Bishop 2012). A principios de los cincuentas, compositores como lanis Xenaquis, Karlheinz Stockhousen y John Cage componen "partituras abiertas" donde el intérprete podía decidir el orden de los movimientos, o donde la participación espontánea del público era requerida. Otro ejemplo son las composiciones donde el azar se constituía como único factor constructivo de la composición. A finales de esta misma década y principios de la década de los sesenta, las prácticas situacionistas, como las derivas psicogeográficas y la emergencia del performance, el happening o la instalación, desarrolladas por artistas como John Cage, Allan Kaprow, Merce Cunningham, Nam June Paik, Yoko Ono, Fluxus, Helio Oiticica, Bruce Nauman, Richard Serra o Joseph Beuys, entre otros (Grant 2011). Propuestas que componían un cuerpo inmaterial de producción artística, que ejercía una traslación de los intereses creativos del objeto al proceso y que a su vez, asumía un posicionamiento político de los artistas ante la autonomía y economía del arte.

En los noventa las estrategias participativas vuelven a hacerse presentes en la cultura mainstream del arte contemporáneo. Pero a diferencia de las prácticas participativas de los sesenta con un marcado carácter activista, ideológicamente situadas, contraculturales y políticas. Las exploraciones en los noventas parten desde un enfoque distinto al incluir entre sus premisas de actuación la emergente relación humano-máquina. La naturaleza híbrida, rizomática, discreta, interactiva y compleja de las computadoras establece nuevas formas 
discursivas, nuevas realidades "virtuales", nuevas relaciones y formas comunicacionales basadas en las tensiones software-hardware, real-virtual, relaciónal-objetual, global-local, etc. y relacionadas directamente a la participación de las audiencias en el diálogo humano-máquina, sujeto-dispositivo-sujeto.

Esta imbricación de los sistemas lógicos y los sistemas sociales produce un escenario nuevo para la acción creativa. Como a principios del siglo pasado, @@s artistas se encuentran ante un nuevo panorama tecno-cultural por explorar; solo que en este nuevo comienzo, no es la incorporación de aparatos y maquinarias a la producción artística lo que genera nuevos lenguajes, estrategias narrativas e imaginarios colectivos. Son las lógicas del software, la parte informática e intangible de la maquinaria, la que incentiva la exploración relacional, comunicacional de las tecnologías. En este sentido las estrategias participativas trabajan conscientemente fuera del espacio del arte con la exploración de estructuras de relación y encuentro, redes de comunicación y creación colectiva.

\section{METODOLOGÍA}

Los trabajos artísticos enfocados a la producción de formas de interacción, participación y relación son muy heterogéneos, las propuestas participativas e interactivas son tan variadas como sus intenciones y sus estructuras de funcionamiento. Los trabajos se trasladan sistemáticamente fuera de los espacios del arte hacia distintas áreas del diseño, informática, biología, política, sociología, matemática, genética, etc. Su único denominador común es la búsqueda estructuras de activación, participación y producción de momentos y espacios colectivos de expresión, diálogo y autoproducción. Por tanto aunque las estrategias participativas pueden ser útiles en la construcción de nuevas formas de relación social, no todos los trabajos de arte participativo tienen dichos objetivos. Para hablar de las posibilidades que esta forma de producción artística puede aportar en términos de regeneración social, disidencia o emancipación nos enfocaremos en proyectos artísticos que hacen uso de las estructuras de participación para crear sinergias sociales e inferir directamente en la realidad de los participantes en el sentido que estos lo decidan, según sus problemáticas y contextos. 
Los proyectos que se utilizan como caso de estudio a continuación, han sido seleccionados, entre muchos otros, dado que responden a un criterio acotado y establecido: son propuestas artísticas que ponen en relación a distintos actores sociales e intentan re-significar las dinámicas de dichos actores con el afán de desarrollar espacios propicios para la emergencia de nuevas dinámicas sociales, nuevos constructos culturales y nuevas soluciones locales a problemas contextuales.

\section{REVISIÓN DE OBRAS}

Es común que éste tipo de trabajos artísticos terminen convirtiéndose en proyectos a mediano y largo plazo. Espacios comunitarios de dinamización social, de producción creativa, espacios multidisciplinares, interdisciplinares, de co-producción, educación y acción micro-emancipativa. En 1994 el proyecto Row Houses fue impulsado por un colectivo de artistas que querían generar cambios a través del arte en el barrio de donde provenían. Éste colectivo consiguió que el ayuntamiento les cediera un bloque de ocho casas pre-construidas para dedicarlas a la exhibición y producción de obras de arte. Con los años, se han ido añadiendo nuevas iniciativas al proyecto, incluyendo un programa de residencia / educación para las madres solteras, programas de educación para los niños de la localidad, una lavandería, una salón de baile para eventos públicos, y espacios de arquitectura experimental en colaboración con el programa de arquitectura de la Universidad Rice. Aunque se asemeja a un centro activista comunitario, Project Row Houses a menudo se define como una obra de arte, una arquitectura interactiva y escultura pública (Finkelpearl 2014).

AVL Ville, por otro lado, es una propuesta del colectivo holandés Atelier Van Lieshout (AVL) que se produjo a lo largo del año 2000 y consistía en la proclamación de lo que ellos denominaron un "Estado libre" en el puerto de Rotterdam, con su propia constitución, moneda y bandera. Era un pueblo utópico, donde la gente podía vivir y trabajar de una manera autárquica; a parte de las unidades habitacionales este micro-estado nación contaba con un gran taller donde se hacían obras de

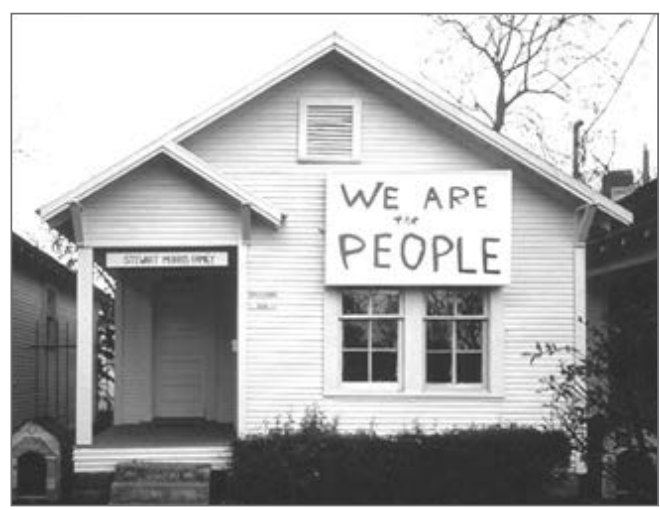


arte, una granja que proporcionaba alimentos y un laboratorio donde se producían alcohol y medicamentos. "Este proyecto proporcionaba una forma de arte no sólo para mirar, sino para experimentar mediante las vivencias cotidianas" (Van Lieshou, 2006). AVL Ville logró mantener una comunidad permanente de 230 participantes y alrededor de dos mil participantes eventuales que mantuvieron viva la villa durante un año, hasta que los integrantes del colectivo AVL decidieron disolver el estado para iniciar nuevos proyectos.

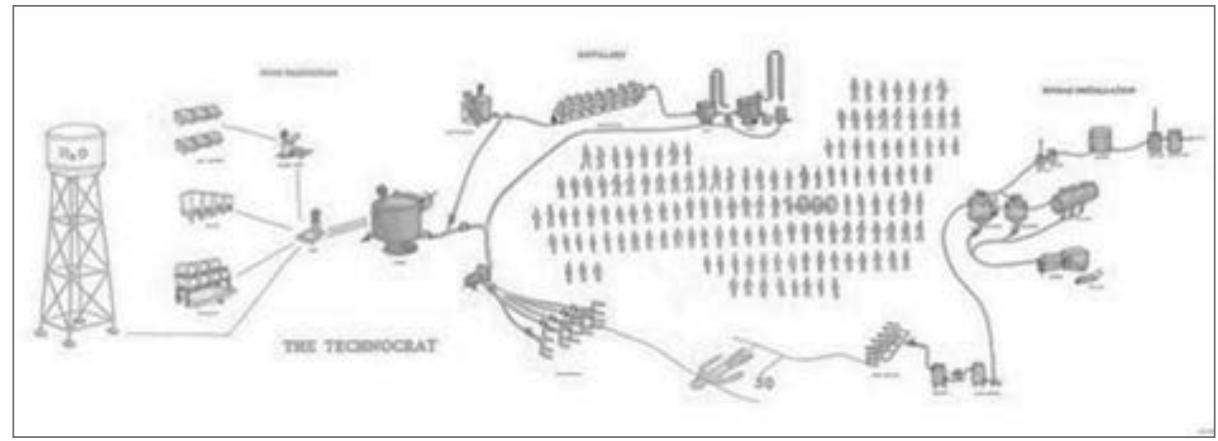

La representación de las cargas policiales que los mineros de Orgreave sufrieron el 18 y 19 de Junio de 1984 fue el origen de La Batalla de Orgreave. El proyecto, ideado por el artista Jeremy Deller, consistió en recrear, como si fuesen batallas medievales, las brutales cargas policiales que soportó el gremio de los mineros en Reino Unido bajo el gobierno de Margaret Tacher. En el juego propuesto por Deller de un lado estaría la comunidad de Orgreave y del otro, los cuerpos policiales representados por colectivos dedicados a la representación de batallas históricas del Reino Unido. La intención del proyecto según Jeremy es la de generar un evento colectivo que hablara de un trauma común y sirviera como una especie de catarsis colectiva para superarlo.

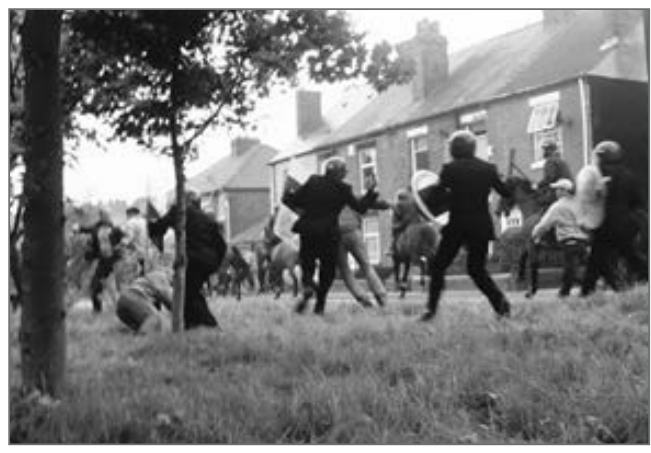

La Escuelita zapatista: en el mes de marzo de 2013 los zapatistas a través de la Comisión Sexta enviaron cartas a más de mil personas de todo el mundo para invitarlos a tomar el curso de Primer Nivel titulado "La Libertad según los zapatistas" a efectuarse del 12 al 27 de agosto de ese año. Los participantes hacían un registro en internet, y se presenciaban en el CIDECI (Centro Indí- 
gena de Capacitación Integral) en San Cristóbal de las Casas donde eran dirigidos a una comunidad para participar de las actividades diarias de una familia asignada para así, de manera directa y vivencial, conocer las estructuras de autodeterminación que se han conformado durante los últimos veinte años de emancipación. A partir del 2013 la convocatoria para participar en la escuelita zapatista se ha mantenido. Cientos de personas de distintas partes del mundo experimentan la forma de vida de las comunidades zapatistas, y en la práctica cotidiana ven las estructuras de autonomía en todas las áreas y de manera colectiva: salud, economía, educación, organización, justicia, cultura, política y gobierno, agroecología y comunicación. En cada una de las áreas buscan mostrar sus valores y formas organizativas confrontándolas con lo que hace el "mal gobierno".
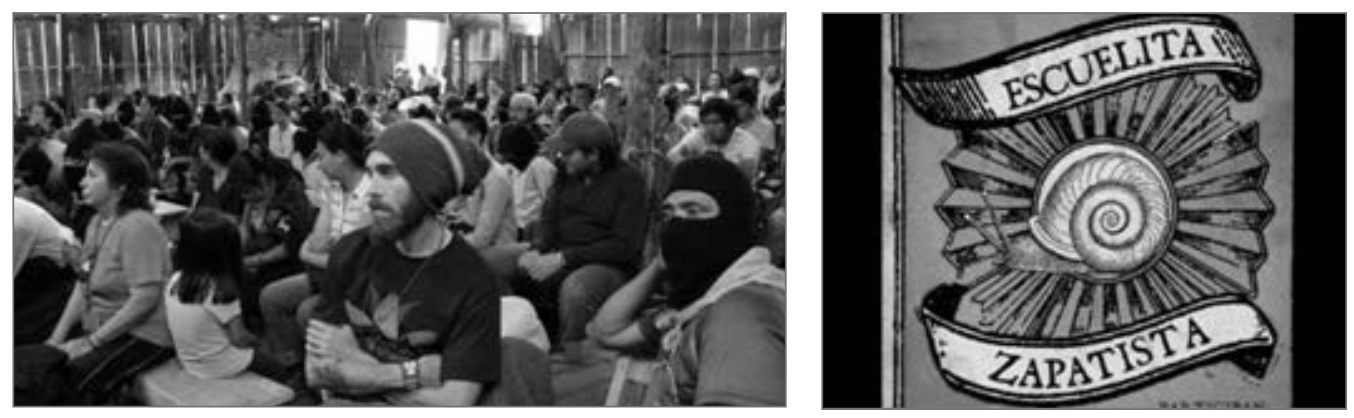

\section{RESULTADOS}

Los trabajos anteriores, se abocan a la producción de estrategias de comunicación y participación comunitaria con la intención de explorar las capacidades del trabajo artístico como nuevo marco de producción de relaciones sociales y políticas. El valor de dichas exploraciones reside en su capacidad para visibilizar eventos que no terminan de suceder. El lenguaje insinuativo y difuso del arte es sumamente eficaz para describir el estado de incertidumbre, heteronomía y complejidad en el que nos hayamos inmersos. "Los artistas se presentan como investigadores y pensadores que desafían en sus trabajos los consensos antropológicos y filosóficos sobre los órdenes sociales, sobre las redes de comunicación y los vínculos entre individuos y sus modos de agruparse" (Canclini 2010, 47). Este tipo de trabajos exploran las relaciones y la interacción, los diálogos y tensiones que emergen de la relación individuo-sistema. Cuando los trabajos artísticos participativos superan la barrera del efectismo y el espectáculo visual, ponen al usuario en relación directa con el dispositivo técnico, muestran el funcionamiento de las estructuras y las ponen a 
su disposición. Subvirtiendo la relación de poder establecida entre sistema e individuo. Por tanto, las estrategias participativas propuestas desde el ámbito artístico trabajan en el desarrollo de "tecnologías blandas" de relación entre individuos o colectivos, proponiendo métodos de participación tecnoculturales, transversales, interdisciplinares y complejos.

\section{DISCUSIÓN}

La creación de estrategias de colaboración y producción colectiva tienen una implicación política implícita. Exploran distintas formas, estructuras y metodologías que permiten la producción de relaciones sociales destinadas a fomentar el pensamiento crítico y la acción emancipatoria. Su producción reafirma los procesos identitarios y desarrolla nuevas formas de relación comunitaria, por tanto es una acción análoga a la micropolítica.

En este sentido parece que la actitud crítica más efectiva en estos momentos sólo puede ser pensada en términos de creación de algo nuevo, como la producción de otros imaginarios. Las practicas participativas contribuyen a la generación de nuevos marcos de relación y en ese sentido expanden las fronteras del arte hacia la creación y emancipación social. Generan métodos, estrategias, dinámicas y estructuras de relación (tecnologías blandas) que pueden ser útiles en la reconstrucción del tejido social. Al mismo tiempo que exploran las estrategias de relación y producción política, fuera del campo de la autonomía del arte actúan como nodos, donde distintas disciplinas y actores sociales convergen se imbrican y reinventan. El artista entonces, actúa como un profesional de la gestión de relaciones; un conductor y mediador que coordina y regula la acción interdisciplinar e intermedial de los procesos interdisciplinares en los que participa.

Las prácticas participativas habilitan nuevas funciones de la producción artística, establecen nuevos nichos de acción en la sociedad y generan nuevos cuestionamientos: ¿qué hacer con aquello para lo que no se encuentran respuestas en la cultura, ni en la política, ni en la tecnología? ¿Cómo articular acciones conjuntas desde la fragmentación y la heteronomía?

No obstante, a pesar del potencial "emancipatorio" de las prácticas participativas, aún existen muchos espacios indiferenciados y poco definidos en 
cuanto a las competencias del arte y su idoneidad en la irrupción en el marco socio-político, ya que para muchos investigadores la esencia difusa, ambigua e insinuativa que da valor de los lenguajes artísticos (abiertos a la múltiple interpretación) es incompatible con la pragmática concreción que requiere la política.

Por otra parte, la praxis artística también debe ser analizada, ya que muchos de los trabajos propuestos no pueden asegurar su continuidad en el tiempo, y terminan siendo anecdóticos, o bien, "objeto de estudio" para historiadores o sociólogos. Los trabajos participativos, en su mayoría de alta intensidad y corta duración, tienen que replantear sus estrategias de acción para ser realmente eficaces en el objetivo de llevar a cabo la transformación contextual propuesta y fomentar la emancipación social.

\section{Referencias}

Bishop, Claire. 2012. Artificial Hells: Participatory art and the politics of spectatorship. London: Verso

Bishop, Claire, ed. 2005. Participation: Documents of contemporary Art. London: Whitechapel

Bourriaud, Nicolas. (1998) 2006. Estética relacional. Traducción de Cecilia Beceyro y Sergio Delgado. Buenos Aires: Adriana Hidalgo. http://es.scribd.com/doc/20953306/Estetica-relacional-Nicolas-Bourriaud

Brea, José Luis. 2007. Cultura RAM: Mutaciones de la cultura en la era de la distribución electrónica. Barcelona: Gedisa

Coulter-Smith, Graham. 2006. Deconstructing Installation Art. Nueva York: Casiad

Eco, Humberto. (1962) 1990. La obra abierta. Traducción de Roser Berdagué. Barcelona: Ariel

Finkelpearl, Tom. 2014. “Participatory Art”. En Encyclopedia of Aesthetics, Michael Kelly, ed. New York: Oxford University. Acceso, 3 dic. 2015, http://arts.berkeley.edu/wp-content/ uploads/2015/03/Participatory Art-Finkelpearl-Encyclopedia Aesthetics.pdf

García Canclini, Néstor. 2010. La sociedad sin relato: Antropología y estética de la inminencia. Buenos Aires: Katz

Gianetti, Claudia. 2007. "Aesthetics and communicative context". Medien Kunst Netz [Media Art Net]. Aesthetics of the Digital Aesthetics/Communication. Acceso 2 jun. 2015, http:// www.medienkunstnetz.de/themes/aesthetics of the digital/aesthetics and communicative\%20Context/scroll/

- 2007. "Aesthetics paradigms of media art". Medien Kunst Netz [Media Art Net]. Aesthetics of the Digital Aesthetics/Communication. Acceso 23 may. 2015, http://www.medienkunstnetz.de/themes/aesthetics of the digital/aesthetics and communicative\%20Context/

- 2007. "Cybernetic aesthetics and communication". Medien Kunst Netz [Media Art Net]. Aesthetics of the Digital Aesthetics/Communication. Acceso 23 may. 2015, http://www. medienkunstnetz.de/themes/aesthetics of the digital/cybernetic aesthetics/ 
Grant, Kester. 2011. The One and the Many: Contemporary collaborative Art in a global context. New York: Duke University

Jin, Zhouying. 2005. Global Technology change: From hard Technology to soft Technology. Bristol: Intellect

Lozano-Hemmer,Rafael. 2012. "Entrevista". PortavozTV. Vídeo de Youtube, 8:22. Publicado el $20 \mathrm{abr}$. https://www.youtube.com/watch?v=|BL6imnolEk\&feature=youtube gdata player

Manovich, Lev. 2005. The Language of New Media. Boston: MIT

Martín Prada, Juan. 2008. "La creatividad de la multitud conectada y el sentido del arte en el contexto de la Web 2.0". "24/7: políticas de la visualidad en un mundo 2.0", número monográfico, Estudios Visuales 5. http://www.estudiosvisuales.net/revista/pdf/num5/prada 20. $\underline{\mathrm{pdf}}$

Romero, Brenda. 2013. "GDC 2013-Interview with Brenda Romero". CologneGameLab. Vídeo de Youtube, 9:25. Publicado el 28 mar. https://www.youtube.com/watch?v=9-MSjiJOoi8

Van Lieshout, Joep. 2006. "In huis bij Joep van Lieshout". Electricalfilms. Vídeo de Youtube, 4:30. Actualizado 10 ene. 2012. https://www.youtube.com/watch?v=rrSICu 5sxU 